\title{
Mobilität(en) - ein neues Paradigma für die Verkehrsgeschichte?
}

Anne-Katrin Ebert

Peter Adey, David Bissell, Kevin Hannam, Peter Merriman, Mimi Sheller (Hg.) 2014: The Routledge Handbook of Mobilities. London und New York: Routledge, geb., 624 S., 140,00 f, ISBN: 978-0-41566771-5.

Peter Merriman 2012: Mobility, Space and Culture. [=International Library of Sociology] London und New York: Routledge, brosch., 214 S., 26,99 f, ISBN: 978-0-415-73698-5.

Gijs Mom 2015: Atlantic Automobilism, Emergence and Persistence of the Car, 1895-1940. New York und Oxford: Berghahn, geb., 768 S., 95,00 f, ISBN: 978-1-78238-377-2.

Christoph Neubert, Gabriele Schabacher (Hg.) 2013: Verkehrsgeschichte und Kulturwissenschaft. Analysen an der Schnittstelle von Technik, Kultur und Medien. Bielefeld: transcript Verlag, brosch., 322 S., 34,80 €, ISBN: 978-3-8376-1092-5.

Anette Schlimm 2011: Ordnungen des Verkehrs. Arbeit an der Moderne - deutsche und britische Verkehrsexpertise im 20. Jahrhundert. Bielefeld: transcript Verlag, brosch., 366 S., 35,80€, ISBN: 978-3-8376-1828-0.

Mimi Sheller 2014: Aluminium Dreams. The Making of Light Modernity. Cambridge, MA und London: MIT Press, geb., 384 S., 20,95 f, ISBN: 978-0-2620-2682-6. 
Richard Vahrenkamp 2011: Die logistische Revolution. Der Aufstieg der Logistik in der Massenkonsumgesellschaft. Frankfurt am Main: Campus Verlag, brosch., 390 S., 39,90 €, ISBN: 978-3-59339215-8.

On the move lautete der programmatische Buchtitel, mit dem der Londoner Sozialgeograph Tim Cresswell seine 2006 veröffentlichte Monographie zur Mobility in the Modern Western World betitelte. Cresswell beschrieb darin unsere Welt der Gegenwart, in der Mobilität zu einem bestimmenden Wert und Merkmal geworden ist. Gleichzeitig verband er mit dem Slogan „on the move" den Appell an seine eigene Disziplin, die Geographie, sich dem Phänomen Mobilität inhaltlich und methodisch zu nähern. Er beklagte die „Leerstelle Mobilität” in seiner Disziplin, die damit ein zentrales Merkmal unserer Welt und der Wahrnehmung unserer Welt übersehe (Cresswell 2006, $1 \mathrm{f}$.$) .$

„On the move" könnte auch der Titel dieses Review-Essays sein, versucht er doch, die gleichermaßen vielfältigen, sowohl ineinander als auch voneinander weg fließenden Bewegungen auf dem Feld der Mobilitätsforschung in den Blick zu bringen. Denn das Schlagwort Mobilität erscheint geradezu allgegenwärtig im wissenschaftlichen Diskurs; es beschreibt Ansätze und Forschungsperspektiven der Geographie und der Soziologie ebenso wie der Literaturwissenschaft, der Medienwissenschaften, der Kulturwissenschaften, der Anthropologie, der Philosophie, sowie der Verkehrs- und Technikgeschichte. Aus all diesen Disziplinen stammen die Autorinnen und Autoren der hier zu diskutierenden Veröffentlichungen, und das gemeinsame, interdisziplinäre Interesse an der Mobilität ist Thema dieses Essays.

Die hier zu diskutierende Literatur ist sowohl in den Themen als auch in den Herangehensweisen recht unterschiedlich; deshalb erscheint es sinnvoll, die jeweiligen Veröffentlichungen zumindest kurz in ihrer Argumentation und ihrer Herangehensweise zu charakterisieren. Zugleich ist dieser Essay vor allem geleitet von einem weiterführenden Interesse an den Potentialen dieses interdisziplinären Wissenschaftsdiskurses für die Technik- und Verkehrsgeschichte im engeren Sinne. Dies führt zu gewissen Ungleichgewichtungen bei der Diskussion der Literatur, denn nicht alle Ansätze und Aspekte können in diesem Rahmen gleichermaßen berücksichtigt werden. Gerade bei den Sammelbänden und Handbüchern war eine Auswahl der Artikel zum Zwecke der Zuspitzung der Argumentation notwendig. Ziel dieses Essays ist es, anhand der hier zu diskutierenden Veröffentlichungen danach zu fragen, was die Ansätze der mobility studies aus dem Blickwinkel der Technikgeschichte zu leisten vermögen und wie sie die Forschung zu Verkehr und Kommunikation bereichern können. Umgekehrt soll anhand der interdisziplinären, historisch orientierten Studien zur Mobilität aber auch diskutiert werden, welchen Beitrag die Technikgeschichte $\mathrm{zu}$ diesem interdisziplinären Feld der 
Mobilitätsforschung leisten kann. Letztlich wird es darum gehen, kritisch zu hinterfragen, wo der tatsächliche Mehrwert einer Mobilitätsgeschichte liegt, ob sich mit dem neuen Begriff tatsächlich neue Ansätze und Methoden verbinden oder ob der Begriff der „Mobilität” womöglich nur handlich bündelt, was zuvor schon mit anderen Begriffen, etwa dem des Verkehrs oder der Warenströme, thematisiert und bearbeitet wurde.

Viele der Autorinnen und Autoren setzen Methoden und Fragestellungen aus den Nachbardisziplinen ein, greifen deren Diskussionen und Ergebnisse auf und tragen so zu einer interdisziplinären Diskussion des Phänomens Mobilität bei. Geographen und Soziologen wie Peter Merriman und Mimi Sheller setzen historische Analysen in ihren Untersuchungen ein, umgekehrt entwickeln Historiker wie Colin Divall und Gijs Mom Plädoyers für eine usable past. Mit diesem aus der amerikanischen Geschichtsschreibung stammenden Begriff verbinden die beiden Autoren den Anspruch, mit ihrer historischen Analyse Politikberatung für die Gegenwart und Zukunft anbieten zu können. Zugleich ist zu betonen, dass trotz der interdisziplinären Ansätze die disziplinären Prägungen in den vorliegenden Bänden nach wie vor deutlich zu spüren sind.

So ist es trotz des vermeintlich gemeinsamen Forschungsinteresses auch ein Wagnis, aus den vielfältigen Stimmen zu Mobilität und Verkehrsgeschichte einen irgendwie zusammengehörigen Chor machen zu wollen. Denn selbst wenn in allen zu diskutierenden Bänden Verkehr und Mobilität Untersuchungsgegenstände bilden und viele von ihnen auf denselben Textkanon der neueren Mobilitätsforschung zurückgreifen, so sind die Grenzen der traditionellen Disziplinen und die Unterschiede zwischen Geistes-, Sozialund Naturwissenschaften bisweilen deutlich spürbar. Akademische Karrieren verlaufen hauptsächlich entlang von Disziplinen, so dass Autorinnen und Autoren vor allem das jeweils eigene Publikum bedienen müssen. Dies gilt insbesondere für die deutschsprachige Forschung. Der Übergang vom vielstimmigen Chor zur Kakophonie ist daher bisweilen fließend.

Doch nicht nur in den disziplinären Orientierungen, auch im Aufbau unterscheiden sich die hier zu diskutierenden Veröffentlichungen erheblich. So gibt es Monographien zu bestimmten Aspekten von Mobilität (Mom 2015; Sheller 2014; Schlimm 2011; Vahrenkamp 2011), theoretische Reflexionen zu Methoden und Erkenntnisinteressen (Merriman 2012), Sammelbände (Neubert and Schabacher 2013) und Handbücher (Adey et al. 2014). Auch hier adressiert der Mobilitätschor unterschiedliche Zuhörerschaften. Während es einigen um die inhaltliche und methodische Weiterentwicklung der interdisziplinären (und historisch orientierten) Mobilitätsforschung geht (Adey et al. 2014; Mom 2015; Sheller 2014), geht es anderen um die „Wiedergewinnung eines Forschungsfeldes" (Neubert and Schabacher 2013: 7), wieder andere verfolgen die Mobilitätsforschung eher von außen und untersuchen das Teilgebiet Verkehr im Rahmen einer Analyse gesamtgesellschaftlicher 
Phänomene wie dem Ordnungsdenken und social engineering (Schlimm 2011). Daher soll zunächst das Gravitationszentrum der mobility studies, also deren Protagonisten, Organisationsformen und Zeitschriften vorgestellt werden, ehe im Folgenden anhand der $\mathrm{zu}$ besprechenden Bücher die Begrifflichkeiten von "Verkehr” und „Mobilität", sowie charakteristische Fragestellungen, Themen und Methoden diskutiert werden.

\section{Mobility Studies im Kontext der Verkehrsgeschichte: Internationale Protagonisten und Foren}

In der englischsprachigen Forschungslandschaft ist die Tendenz zur Interdisziplinarität in der historischen Forschung zur Mobilität deutlich stärker ausgeprägt. So haben sich die Inhalte des Journal of Transport History, das sich seit über fünfzig Jahren dem engeren Feld der Verkehrsgeschichte widmet, in jüngerer Zeit deutlich gewandelt, die Perspektive auf Nutzer/innen und Konsumenten hat die zuvor stark dominierende Untersuchung der Verkehrsträger ergänzt. ${ }^{1}$ Daneben hat sich eine weitere Zeitschrift mit dem programmatischen Namen Transfers. Interdisciplinary Journal of Mobility Studies etabliert. Mit dem zusätzlich erscheinenden Jahrbuch Mobility in History verfügt das einstige Nischenfeld Verkehrsgeschichte nunmehr über drei internationale Periodika, die wiederum alle drei auf unterschiedliche Art und Weise mit der 2003 gegründeten internationalen Vereinigung T2M International Association for the History of Transport, Traffic and Mobility verbunden sind. ${ }^{2}$ Mehrere Autorinnen und Autoren der hier zu diskutierenden Bände stehen in einem engen Verhältnis zu T2M und den oben genannten Diskussionsforen; Gijs Mom war der Gründer und langjährige Präsident von T2M, Mimi Sheller ist die gegenwärtige Präsidentin. ${ }^{3}$

Sheller, Direktorin des Center for Mobilities Research an der Drexel University, ist zugleich eine Mitbegründerin der mobility studies. Gemeinsam mit John Urry, dem Direktor des Centre for Mobilities Research an der Universität Leicester, rief sie im 2006 erschienen Artikel „The New Mobilities Paradigm" - der inzwischen zusammen mit Cresswells im gleichen Jahr erschienener Monographie On the Move zu den kanonischen Texten für das noch junge Forschungsgebiet zählt - dazu auf, die sozialwissenschaftliche Erforschung von Mobilitäten zu intensivieren (Sheller and Urry 2006). Mit der von Urry, Sheller und Kevin Hannam mitinitiierten, 2006 erstmals erschienenen Zeitschrift mobilities hat dieser stärker gegenwartsbezogene, sozialwissenschaftliche Ansatz der Mobilitätsforschung ein eigenes Forum, dessen Methoden- und Inhaltsdiskussion maßgeblich die historische Forschung beeinflusst (Hannam et al. 2006). In seiner im darauffolgenden Jahr publizierten Monographie gleichen Namens untermauerte Urry sein Plädoyer 
für neue Ansätze in den Sozialwissenschaften noch einmal ausführlich (Urry 2007). Der Einfluss dieser sozialwissenschaftlichen Diskussion lässt sich in den meisten der hier zu diskutierenden Bände aufzeigen, und eine Bezugnahme darauf gehört bei fast allen zum guten Ton. Nur in einer einzigen deutschsprachigen Arbeit wird kein einziger dieser Autoren rezipiert (Vahrenkamp 2011).

\section{Mobilität und Verkehr: Ein Annäherungsversuch an die beiden dominierenden Begriffe}

Trotz der mittlerweile gängigen Verbindung des Begriffs Mobilität mit den Forschungsprogrammen von Creswell, Sheller und Urry sei an dieser Stelle noch einmal ausdrücklich daran erinnert, dass insbesondere in der deutschsprachigen Forschung der Begriff Mobilität in der Verkehrsgeschichte sich nicht zwingend auf diese englischsprachigen Forschungszweige bezieht. Noch 2008 benutzte der Schweizer Historiker Christoph Maria Merki in seinem Handbuch Verkehrsgeschichte und Mobilität eben jenes Schlagwort ohne Rückgriff auf die zwei Jahre zuvor erschienen Werke von Cresswell, Sheller und Urry. Merki führte den Begriff „Mobilität“ ein, um die Forschungsperspektiven über eine Geschichte der Verkehrsträger hinaus hin zu soziokulturellen Fragestellungen nach der Entstehung von „Mobilitätsbedürfnissen” zu erweitern. Er plädierte für eine „multiperspektivische und interdisziplinäre Sichtweise" (Merki 2008: 111), die Produktion und Nutzung gleichermaßen berücksichtigt und auch nach der historischen Bedingtheit von Mobilitätsbedürfnissen fragt (Merki 2008: 115).

Zugleich wies bereits Merki auf die unterschiedlichen Bedeutungsgehalte der deutschen und der englischen Sprache hin, da der deutsche Begriff „Verkehr” ein wesentlich größeres Bedeutungsspektrum einzunehmen vermag als das englische Pendant „transport” (Merki 2008: 9). Aus eben diesem Grund brechen die Medienwissenschaftler Christoph Neubert und Gabriele Schabacher in ihrer lesenswerten, da einen kompakten Einblick in aktuelle Fragestellungen liefernden Einleitung zu ihrem Sammelband Verkehrsgeschichte und Kulturwissenschaft eine Lanze für den Begriff „Verkehr”. Dieser, darauf weisen Neubert und Schabacher zu Recht noch einmal deutlich hin, verfügt über das Potential, die schon von Merki und anderen Verkehrshistorikern beklagte Verengung auf die Geschichte einzelner Verkehrsträger aufzubrechen. Denn erst im 19. Jahrhundert entwickelte sich die Unterscheidung des Verkehrs in seine Transportfunktionen für Personen, Waren und Nachrichten. Neubert und Schabacher erinnern dagegen an den weiten historischen Bedeutungshorizont des Wortes „Verkehr”, dessen Semantik „gesellschaftlicher Verkehr, Umgang, Geselligkeit sowie Handels- und 
Warenverkehr” umfasst, sowie das Verb „verkehren”, das sich auf Prozesse der „Umkehrung, Verwechslung und Verwandlung” bezieht (Neubert and Schabacher 2013: 22). Der Rückgriff auf diese vormodernen Semantiken ermöglicht nicht nur die Revision der Trennung von Verkehrs- und Nachrichtensystemen, sondern auch die Aufhebung jener spezifisch modernen Trennung zwischen Sphären materieller, sozialer und symbolischer Prozesse (Neubert and Schabacher 2013: 23). Gerade letzteres macht den Begriff Verkehr für die Medienwissenschaftler Neubert und Schabacher attraktiv.

Von Verkehr spricht auch Annete Schlimm in ihrer Dissertation zu den Ordnungen des Verkehrs, der einzigen diskursgeschichtlichen Analyse in der hier zu diskutierenden Literaturauswahl. Dabei zeigt gerade ihre präzise Analyse des verkehrlichen Ordnungsdenkens in Deutschland und England, wie gewinnbringend die Diskursanalyse auch für die Verkehrsgeschichte sein kann. Schlimms Augenmerk richtet sich auf die diskursiven Mechanismen, vermittels derer sich Verkehrsexperten in Deutschland und England zwischen den 1920er und 1950er Jahren als Experten etablierten und legitimierten. Im Kontext einer dynamischen Moderne, die die etablierte Ordnung zu zerstören drohte, beschworen die Verkehrsexperten „Verkehr” als ein zusammenhängendes, einerseits physisch-technisches, andererseits aber auch immaterielles, soziales Gebilde, das den harmonischen Ausgleich zwischen lokalen und nationalstaatlichen Verkehrsebenen, sowie Dynamik und Statik in einer quasi überzeitlichen, mithilfe moderner Wissenschaft hergestellten Ordnung gewährleisten sollte. Verkehr wurde so zu einem zentralen Bereich der Gesellschaftsgestaltung.

In Anlehnung an Foucault betont Schlimm die mit der Episteme „Verkehr" einhergehenden Machtverhältnisse, die sich nicht nur auf das Verhältnis von Experten und Laien bezogen, sondern auch auf die Vorrangstellung der „vermeintlichen sozialen Gesamtheit gegenüber individuellen Vorlieben und Interessen” (Schlimm 2011: 308). Es gab „richtigen” und „falschen” bzw. „notwendigen” und „unnötigen” Verkehr. In den Augen der Experten war dieser nur dann zulässig, wenn er der Allgemeinheit diente. Wenn er hingegen aus partikularen, individuellen Interessen erwuchs, war er überflüssig. Abschließend charakterisiert Schlimm diese Phase der deutschen und britischen Verkehrsexpertise in den 1920er und 1950er Jahren als ,antiliberale[n] und antipartizipatorische[n] Versuch, eine Ordnung des Sozialen über die Position des Experten zu implementieren - mit einem starken Staat im Hintergrund und ohne eine demokratische Aushandlungsmöglichkeit" (Schlimm 2011: 312). Zugleich regt Schlimm eine Historisierung des Begriffs Mobilität an, den sie als Konzept der Nachkriegszeit in den Debatten über Konsumgesellschaft und Modernisierung der 1960er Jahre verortet (Schlimm 2011: 299). Mobilität bezeichnet in diesem Zusammenhang die dezidierte Hinwendung zu den Wünschen der Verkehrsnutzer: Verkehr wird zum Konsum, und die freie, unreglementierte Verkehrsnutzung 
wird zum Rückgrat wirtschaftlicher Prosperität und demokratischer Ordnungsprinzipien.

Zeigen Neubert und Schabacher in ihrer Einleitung auf, welche Potentiale im semantischen Feld des Begriffs „Verkehr” stecken, so lässt sich Anette Schlimms Monographie als Hinweis darauf lesen, warum sich der Begriff „Verkehr” angesichts seiner zunehmenden Verengung und Einbindung in die Expertendiskurse in der Mitte des 20. Jahrhunderts eben gerade nicht mehr für eine wissenschaftliche Mobilisierung in der Folgezeit anbot. Außerdem ist der Hinweis auf den engen Zusammenhang von Mobilität und Konsumgesellschaft ein wesentlicher Aspekt, der auch in den anderen, hier noch zu diskutierenden Veröffentlichungen immer wieder auftaucht.

Der Begriff Mobilität ist in der deutschsprachigen Verkehrsgeschichte ebenso wie in der englischsprachigen transport history - verwendet worden, um das bisweilen geradezu hermetisch entlang der unterschiedlichen Verkehrsträger Schiene, Schifffahrt, Straße und Luftfahrt organisierte Feld wieder zusammenzuführen. Zugleich verbindet sich mit dem Schlagwort Mobilität der Wunsch nach einer erneuten Zusammenführung der auseinanderdividierten Transporteinheiten Personen, Waren und Nachrichten. Drittens beschreibt Mobilität die Umorientierung von der Produktion von Verkehr hin zum Konsum. Nicht zuletzt verknüpften sich mit dieser Umorientierung auch sozial- und kulturhistorische Fragestellungen, die die bis dato dominierenden sozial- und wirtschaftshistorischen Ansätze teilweise ablösten.

In der Forschungspraxis wird der Begriff Mobilität nach wie vor auf die moderne Geschichte, insbesondere das 20. Jahrhundert, bezogen. In dieser Hinsicht überwiegt der deskriptive Charakter, der Mobilität und Moderne in enger Wahlverwandtschaft sieht. Gleichzeitig hatte insbesondere John Urry eben gerade nicht die gegenwärtige Welt als mobiler als vergangene Welten beschreiben wollen. Vielmehr misstraute er grundsätzlich der vermeintlichen Tendenz der Soziologie, mit ihrem zentralen Begriff von der "Gesellschaft” Sesshaftigkeit vorauszusetzen und damit eine gewisse Statik zu implizieren. Ihm ging es vor allem darum, „Mobilität” als relationales Paradigma einzuführen, um das sich eine Forschungsagenda zu komplexen Systemen, Assemblagen und Mobilitätspraktiken gruppiert.

\section{Mobilitäten und Immobilitäten in der Moderne - globale Warenströme}

In ihrer Studie Aluminum Dreams differenziert die Soziologin Mimi Sheller das Bild von der mobilen Moderne, indem sie sowohl die „bright side”, als auch die „dark side” der Geschichte dieses Metalls präsentiert (Sheller 2014: 27). Shellers Untersuchung ist eine Kombination von Technik-, Umwelt-, 
Wirtschafts- und Kulturgeschichte, bei der sie den Aufschwung von Aluminium als Traum von Leichtigkeit, Geschwindigkeit und Modernität, sowie die Entwicklung moderner Mobilitätsinfrastrukturen im globalen Norden mit der gleichzeitigen Implementierung von „regimes of immobility” im globalen Süden konfrontiert. Zugleich ist das Buch ein leidenschaftliches und zutiefst politisches Plädoyer an die Leserschaft vor allem im globalen Norden, das eigene Konsumverhalten und die hohe kulturelle Wertschätzung von Mobilität, Leichtigkeit und Geschwindigkeit zu hinterfragen. Sheller zeigt auf, wie sich das „speed metal” Aluminium mit einer Kombination von neuen Technologien, neuen Ästhetiken und neuen Mobilitätspraktiken vom teuren Material der Kriegsindustrie zu einem Material der Spätmoderne wandelte, mit dem Leichtigkeit und Geschwindigkeit verbunden waren. Sie verweist auf die Bedeutung von stromlinienförmigen Leichtbaumodellen für Busse, Züge und Schwerlastkraftwagen, die nicht nur auf den speziellen Materialeigenschaften von Aluminium aufbauten, sondern auch eine „vision of mobility” (Sheller 2014: 90) mit dem Metall verbanden. Dabei hat das Material, wie Sheller selbst konstatiert, seit Ende der 1960er Jahre viel seines einstigen Glamours verloren und wird zusehends mit billigem Häuserbau und Trailerparks assoziiert.

Trotz dieser historischen Veränderungen basiert die Buchstruktur stark auf jener anfänglichen Dichotomie zwischen Leichtigkeit, Mobilität und Hightechdesign einerseits sowie Schwerindustrie, Krieg, Vertreibung und wirtschaftlicher Zurückgebliebenheit der Tropen andererseits. An den ersten Teil des Buches über den Aufstieg des Aluminiums als „speed metal” im globalen Norden schließt ein zweiter Teil über die Folgen dieses Erfolges für den globalen Süden an. Dieser Wechsel von der „bright side” zur „dark side” erfolgt mit einem Kapitel über die Karibikkreuzfahrten des US-Aluminiumherstellers Alcoa. Die Alcoa Shipping Company transportierte nicht nur Bauxit aus der Region ab, sondern etablierte über ihre Aktivitäten im KreuzfahrtTourismus - die Fahrten selbst, sowie das vielfältige Material, mit dem sie beworben wurden - auch eine „Grammatik der Differenz”, bei der die Karibik als farbenprächtige exotische Landschaft mit fröhlichen Menschen konstruiert wurde. Mit den Bildern von der traumhaften Landschaft verschleierte Alco den tatsächlichen Raubbau an der Natur, sowie die Enteignungen und Vertreibungen, wie sie beispielsweise in Surinam mit dem Bau des BrokopondoStaudamms zur Deckung des enormen Strombedarfs in der Verarbeitung des Bauxits einhergingen. Eindringlich schildert Shelley die bis heute andauernden Bemühungen der damals vertriebenen Saamaka Maroons um internationale Anerkennung, dass das damals annektierte Land ihnen unrechtmäßig genommen wurde (Sheller 2014: 151-157).

Diese Differenz wirkt laut Sheller vor allem auf der diskursiven Ebene mit der Zuschreibung von Mobilitäten. Die traumhafte Landschaft der Karibik erscheint immobil, doch gerade für die Maroons, die sich als Nachfahren 
geflüchteter Sklaven über Jahrhunderte hinweg eine Existenz und eigene Kultur im Urwald gegen die Plantagengesellschaft aufgebaut hatten, brachten die mit dem Bauxit-Abbau einhergehenden Veränderungen eine geradezu ungeheure Mobilisierung mit sich: als Truppen im surinamischen Bürgerkrieg auf der Seite des Rebellenführers Ronnie Brunswick im Kampf gegen die Zentralregierung von Desi Bouterse, als Bürgerkriegsflüchtlinge in den Grenzgebieten Französisch-Guyanas, als wachsende Arbeiterschicht im Niedriglohnsektor in der Hauptstadt Paramaribo.

Die Grammatik der Differenz des globalen Nordens spiegelt nicht die tatsächlichen, sozialen und physischen Bewegungen wider. Gleichzeitig schaut Sheller jedoch kaum darauf, wie die Maroons die Veränderungen in ihrem Leben und ihrer Umgebung darstellen. Auch Surinam kennt mittlerweile motorisierte Einbäume, die die Fahrt die Flüsse hinauf in den Urwald verkürzen, Flugfelder in den entlegenen Teilen des Urwalds, neue Straßen mit einer erstmaligen Verbindung des Landesinneren mit Brasilien, den Aufbau eines Mobilfunknetzes mit Sendemasten im Regenwald. Welche Interpretationen haben die Maroons, dieses in der Grammatik der Differenz als sesshaft konstruierte Volk, das sich tatsächlich durch die vielfältigsten Mobilitäten konstituierte, bezüglich ihrer eigenen Existenz? Das Wertepaar des globalen Nordens mit seiner Unterscheidung zwischen Bewegung/Mobilität/Fortschritt einerseits, sowie Stillstand/Immobilität/Rückstand andererseits demonstriert Sheller anschaulich, andere Bewertungen von Mobilität untersucht sie leider kaum.

Shellers Studie erinnert in ihrer transnationalen Anlage, ihrer Ausgangsbasis in der Karibik und mit ihrem Blick auf Orte der Produktion und der Konsumtion von Aluminium an die nunmehr klassische Globalgeschichte des Zuckers des US-amerikanischen Anthropologen Sidney Mintz (Mintz 1985). Die Parallele zu Mintz wird nicht nur im Klappentext von der Historikerin Joyce E. Chaplin gezogen, auch Sheller selbst stellt diese her (Sheller 2014: 149). Für Historikerinnen und Historiker ist dieses Vorbild kaum überraschend; Mintz' einflussreicher Studie sind mittlerweile eine ganze Reihe von Globalgeschichten einzelner Waren und Güter gefolgt. Insofern bietet Shellers - sehr lesenswertes - Buch Aluminum Dreams womöglich aus Sicht der Soziologie, weniger jedoch aus Sicht von Anthropologie und Geschichtswissenschaft einen wirklich neuen Ansatz für die mobility studies, in deren Kontext sich Sheller dezidiert einordnet (Sheller 2014: 9). Shellers Studie ist jedoch vorbildhaft in ihrer Art, Mobilität und Immobilität zusammenzudenken. Sie kann in aller Deutlichkeit aufzeigen, wie auf der diskursiven Ebene Konfigurationen der Mobilisierung und des Aufbruchs immer auch mit Ausschlussmechanismen und Zuschreibungen von Immobilität verbunden waren. Das Gegensatzpaar Mobilität und Immobilität funktioniert bei ihr allerdings vor allem auf der symbolischen Ebene. Der Nexus zu den tatsächlichen Warenströmen, zu den physischen Mobilitäten von Waren und 
Personen und Informationen, ist eher schwach und vor allem einseitig auf den globalen Norden ausgerichtet. Shelley untersucht kaum, welche Formen der Aneignung und Deutung von Aluminium es vor Ort, in den Ländern des Bauxit-Abbaus, gab. Damit bleibt ungeklärt, inwiefern das Gegensatzpaar Mobilität-Immobilität vor allem ein Konstrukt des globalen Nordens ist. Gäbe es Denkfiguren jenseits dieser Dichotomie?

Während die symbolische Ebene, die Produktion von Bildern und Vorstellungen, ein wichtiger Aspekt von Shellers Analyse der globalen Warenströme beim Aluminium bildet, bleibt Richard Vahrenkamp mit seiner historischen Studie Die logistische Revolution vorwiegend auf der operativen Ebene. Die bei Sheller so wichtige doppelte Sicht auf Gewinner und Verlierer des Warenflusses, spielt bei ihm nur ganz am Rande eine Rolle. Lediglich am Ende seiner Ausführungen vermerkt er knapp, dass die von ihm gezeichnete, scheinbar klassische Erfolgsstory mit einem Widerspruch von „hoher Qualität der Erbringung der logistischen Dienstleistungen und niedrigen Löhnen" einhergeht (Vahrenkamp 2011: 340).

Als emeritierter Professor für Logistik und Wirtschaftswissenschaften an der Universität Kassel ist Vahrenkamp einer späteren Generation jener Gruppe von Verkehrsexperten zuzurechnen, die im Mittelpunkt von Schlimms eingangs diskutierter Studie standen. Auf bisweilen irritierende Weise changiert Vahrenkamp in seinem Buch zwischen historischer Analyse und Axiomen, die aus einem Logistik-Handbuch stammen könnten. Die Geschichte der Logistik betrachtet er vor allem aus einem wirtschaftswissenschaftlichen Blickwinkel. Vahrenkamp will aufzeigen, „wie sich die logistischen Dienstleistungen parallel zur Massenkonsumgesellschaft seit der Jahrhundertwende 1900 entwickelt haben" (Vahrenkamp 2011: 9). Doch trotz des vielversprechenden Titels, der den Blick auf globale Zusammenhänge erwarten lässt, ist seine Studie eigentlich eine Beschreibung logistischer Unternehmensstrukturen im Kaiserreich, der Weimarer Republik und in West-Deutschland. Den physisch-materiellen Hintergrund nimmt er nur am Rande, vor allem als Gegensatz zwischen den Verkehrsträgern LKW und Eisenbahn, in den Blick. Die wachsende Literatur, auch im deutschsprachigen Raum, zu Themen wie Standardisierungen, Container und Paletten, nimmt Vahrenkamp kaum zur Kenntnis; ${ }^{4}$ dem Zusammenhang von Logistiksystemen und Informationstechnologien - der wie ein Damokles-Schwert über seinem Untersuchungsgegenstand schwebt - widmet er sich kaum (Vahrenkamp 2011: 340). Im Mittelpunkt steht die Konkurrenz der Systeme LKW und Eisenbahn, bei der Vahrenkamps ganze Abneigung den verkehrspolitischen Maßnahmen zur Regulierung des LKW-Fernfahrverkehrs und zum Schutze der Eisenbahn der Nachkriegszeit gilt. Vor dem Hintergrund von Anette Schlimms Studie erkennen wir hier einen Verkehrsexperten, der anders als seine Vorgänger im Sinne der neoklassischen Theorie sein vollstes Vertrauen in die Kräfte des Marktes setzt und dessen Sympathie dem LKW-Gewerbe gilt, 
dessen wilde Anfänge in den 1920er Jahren Vahrenkamp ausführlich nachzeichnet (Vahrenkamp 2011: 74-121).

Umso verblüffender ist es, dass der Autor der eigentlichen „logistischen Revolution", die er in den 1990er Jahren verortet, kaum 30 Seiten widmet (Vahrenkamp 2011: 303-334). Hier listet Vahrenkamp zwar viele Faktoren auf - die Liberalisierung von Märkten, die Entstehung eines europäischen Binnenmarktes, der Zusammenbruch des Ostblocks, flexible Fertigungstechniken und erhöhte Typenvielfalt, Just-In-Time-Produktionskonzepte, Standortverlagerung außerhalb Europas, insbesondere nach Asien, die Dislozierung von Produktionsstandorten und deren Integration in Logistikketten, das Entstehen des Supply-Chain-Managements, die Intensivierung des Containerumschlags insbesondere in den Importhäfen, sowie der internetgestützte Informationsaustausch - historisch analysieren tut er diese jedoch nicht.

Von anderer Seite ist daher der Vorschlag eingebracht worden, Vahrenkamps Beitrag weniger als historische Studie denn als Quelle zur Güterverkehrspolitik in Deutschland zu lesen (Dommann 2012). Dieser gleichermaßen maliziöse wie nicht ganz unberechtigte Vorschlag verdeutlicht schlaglichtartig noch einmal die in Deutschland nach wie vor existierenden engen Verflechtungen zwischen Verkehrswissenschaften und Verkehrswirtschaft einerseits sowie Verkehrsgeschichte andererseits. Zugleich aber offenbart er die gewaltigen Herausforderungen, vor denen die moderne Verkehrs- und Mobilitätsgeschichte steht. Die Geschichte der Logistik, verstanden als globale Bewegung von Gütern, die eben nicht nur von Politikern geplant und von Verkehrswissenschaftlern gesteuert wurde, sondern bei der viele unterschiedliche Akteure auf verschiedenen Ebenen agierten, es zu systemischen Verzahnungen, aber eben auch zu Ausschlüssen und Blockaden kam, steckt nach wie vor noch in ihren Anfängen. So wie bei anderen Themen der Geschichtsschreibung werden diese Anfänge von Experten aus der Branche betrieben. Von einer einordnenden Bewertung der Entwicklungen gegen Ende des 20. Jahrhunderts - von Vahrenkamp bereits als „logistische Revolution" ausgerufen - sind wir indes noch weit entfernt.

\section{Auf den Leib geschrieben: Mobile Körper und das Automobil}

Sind Vahrenkamp und Sheller in der klassischen Unterteilung von Verkehr in Waren- und Personenverkehr eher ersterem zuzurechnen, so interessieren sich die folgenden Autoren eher für den zweiten Themenbereich. Peter Merriman lehrt Human Geography an der Aberystwyth University in Wales und attackiert mit seinem Buch Mobility, Space and Culture zwei Fundamente seiner wissenschaftlichen Disziplin, nämlich jene von Zeit und Raum. Mit diesem Vorhaben steht Merriman nicht alleine, vielmehr reiht er sich mit 
seinem Text in eine Reihe prominenter Vorgänger ein, die - wie der eingangs zitierte Tim Cresswell - die Humangeographie noch stärker in Richtung einer handlungsorientierten Disziplin ausrichten wollen. ${ }^{5}$ Sein Buch gliedert sich in zwei Teile, deren Übergänge etwas abrupt daherkommen: In einem theoretisch orientierten ersten Teil argumentiert Merriman, warum die Konzepte von „timespace” und „place/placelessness” zugunsten einer Wissenschaft von "movement” aufzugeben seien. Im zweiten Teil seiner Arbeit sucht er diese Wissenschaft von „movement” exemplarisch am Beispiel des Automobils zu demonstrieren. Das Automobil, so versucht Merriman anhand des frühen Automobilismus in England zu demonstrieren, hat die Wahrnehmung von Zeit und Raum nachhaltig verändert.

Im ersten Teil seiner Abhandlung verdeutlicht Merriman zunächst, wie wichtig die Kategorie „space” für die Geowissenschaften war und dass sie tendenziell die Kategorie "time” überschattet habe, die hingegen eher in den Geschichtswissenschaften triumphierte. Seine weit ausholende, auf zahlreiche Philosophen und sozialwissenschaftliche Theorien rekurrierenden Ausführungen wirken teilweise recht bemüht und lesen sich für den Außenstehenden denn auch eher als Zeugnis dafür, wie stark die Verankerung der Geographie in den Naturwissenschaften wohl sein mag, dass sozialwissenschaftlich orientierte Ansätze ihr eine solche Fülle an Denkern und Ansätzen entgegensetzen müssen.

Dabei haben zahlreiche Geographen diese Zeitlosigkeit bereits kritisiert und zugleich das Verhältnis von Zeit und Raum neu reflektiert. Besagter Tim Cresswell ist ein Exponent dieser Neuinterpretation des Verhältnisses von Zeit, Raum und Bewegung. Merriman treibt dessen Argumentationen auf eine neue Spitze, wenn er erklärt: „Movement is not secondary to the events of spacing and timing. Movement is primary" (Merriman 2012: 43). Zugleich warnt Merriman eindrücklich vor einer Nivellierung der Unterschiede in Bewegungen. Vielmehr fordert er eine präzise Analyse der unterschiedlichen Formen, Arten, Ausdrücke, Repräsentationen und Erfahrungen von Bewegung. Bewegung ist bei Merriman gleichermaßen allgegenwärtig wie vielfältig und komplex, aus der Bewegung heraus konfigurieren sich Orte und Räume ebenso wie Zeiten.

In seiner daran anschließenden historischen Analyse konzentriert sich Merriman vor allem auf britische Quellen der Frühzeit des Automobilismus bis ca. 1910. Merriman ist von Gijs Mom, dessen gewichtige Studie Atlantic Automobilism zumindest teilweise den gleichen Zeitraum abdeckt, stark kritisiert worden (Mom 2013/2014). Der Technikhistoriker Mom warf dem Humangeographen Merriman, bei allem Respekt für dessen historische Quellenarbeit, eine gleichermaßen oberflächliche wie unsaubere Analyse des Phänomens des frühen Automobilismus vor. Tatsächlich übernimmt Merriman viele Behauptungen unkritisch aus der damaligen Motorpresse. Da er die Kontextualisierung und Einbettung der Quellen vernachlässigt, akzeptiert er 
unhinterfragt viele Äußerungen seiner Quellen als Fakten. Ein Beispiel dafür ist sein Argument, das Automobil sei 1907 bereits ein praktisches Verkehrsmittel gewesen und darum habe es ein zunehmendes Interesse für Fragen des Komforts und des Zubehörs gegeben. Merriman übernimmt diese Behauptung aus einem Werbeartikel für Zubehör, ohne Rücksicht auf dessen diskursive Funktionen und ohne auch nur einen Blick auf die tatsächlichen Einsatzgebiete und die Verbreitung des Automobils in dieser Zeit zu werfen (Merriman 2012: 91).

Problematisch an seiner Fallstudie ist außerdem das skizzierte Verhältnis von Maschine und Mensch. Als eine Art deus ex machina erscheint das Automobil auf der Szenerie, konfrontiert den - zwar männlich und weiblich gedachten, aber ansonsten erschreckend unhistorischen und wenig ausdifferenzierten - menschlichen Körper mit einem neuartigen Ensemble an Tätigkeitsanforderungen, Materialien, sozialen Etiketten und subjektiven Empfindungen und Wahrnehmungen. Weder die unterschiedlichen Ausformungen des Automobils in dieser Frühzeit - vom Dampfwagen über das Benzin- bis zum Elektrofahrzeug - noch die unterschiedlichen Akteursgruppen, die Automobil-Enthusiasten und Automobil-Gegner, auf dem Land wie in der Stadt, in der einfachen Bevölkerung genauso wie unter den Gesetzesgebern, kann Merriman adäquat fassen. Vor allem aber konzipiert Merriman Erfahrung viel zu passiv als von außen angestoßene Reaktion auf das Automobil und übersieht dabei komplett deren konstruktiven Charakter als zielgerichtete und selbstverortende Wahrnehmung, die sowohl diskursiv als auch materiell hergestellt wird.

Merrimans historischer Teil über die Veränderung der Wahrnehmung von Zeit und Raum durch das Automobil ist ein erster Entwurf und Fingerzeig, in welche Richtung er zukünftige humangeographische Untersuchungen lenken möchte. Ganz anders angelegt ist hingegen die umfangreiche Monographie seines Kritikers Gijs Mom, der den gleichen Zeitraum mit inhaltlich ähnlichen Fragestellungen untersucht, aber hinsichtlich des Untersuchungszeitraums und -gebiets weit über Merrimans Skizze hinausgeht. Moms Atlantic Automobilism beginnt fast schon ein wenig pompös mit dem Hinweis, das vorliegende Werk sei ,the work of a lifetime” (Mom 2015: xiii). Die fast 800 eng bedruckten Seiten stellen nur den ersten Teil einer auf zwei Teile angelegten Studie der Geschichte des Automobils dar, endet sie doch mit dem für deutsche Verhältnisse ungewöhnlichen, im niederländischen Kontext gebräuchlicheren Jahr 1940. Eine Darstellung der weiteren Kriegsjahre, vor allem aber der Nachkriegszeit beziehungsweise der zweiten Hälfte des 20. Jahrhunderts steht noch aus. Damit deckt auch Moms Atlantic Automobilism jene Zeitspanne des Automobilismus ab, die bis jetzt nach wie vor am besten erforscht ist.

Moms Buch ist als Synthese konzipiert, die die deutsche, niederländische, französische und anglo-amerikanische Forschungsliteratur zum Automobilismus zusammenführt und zugleich mit einer eigenständigen 
Analyse von literarischen und filmischen Quellen zum Automobil ergänzt und konfrontiert. Der Autor beeindruckt mit seinem Überblick über den Stand der Diskussion zur Geschichte des Automobils in den vier oben genannten Sprachen. Seine Meinungen sind dezidiert, manchmal polemisch, und hin und wieder sind Moms Analogien zumindest gewagt. Die Tiefe der Auseinandersetzung mit der Forschungsdiskussion wirkt sich bisweilen negativ auf den Erzählfluss aus, zumal Mom seine auf der Analyse literarischer Quellen aufbauende Kapitel immer an seine synthetisierenden Themenkapitel anschließt.

Trotz der beeindruckenden Fülle an Material und Forschungsdiskussionen bleibt kritisch zu hinterfragen, ob der Titel des Buches Moms eigener Analyse gerecht wird. War der Automobilismus bis 1940 tatsächlich ,atlantisch" oder beschreibt dies nicht vielmehr die von Nachkriegsordnung und Kaltem Krieg geprägte Ausrichtung der heutigen Forschungsdiskussion, die Mom so umfassend rezipiert? Ist es berechtigt, weite Teile Süd-, Ost- und Nordeuropas, zwar in Anführungszeichen, aber dennoch als „europäische Peripherie” zu bezeichnen? Kann der Begriff „atlantisch” mit seinen geopolitischen Assoziationen adäquat ein urbanes Phänomen beschreiben, das lange Zeit von einer weltweit vernetzten sozialen Elite getragen wurde? Mom kann aufgrund seiner umfassenden Kenntnis der Forschungsdiskussionen zwar immer wieder andere, von ihm nicht näher untersuchte Länder und Regionen thematisieren, aber ob dort die Verbreitung des Automobils tatsächlich so grundsätzlich anders verlief, vermag er aufgrund des fehlenden systematischen Vergleichs kaum zu sagen (Mom 2015: 642). Vielleicht wäre es hier trotz der verlockenden Alliteration doch besser gewesen, ein anderes Adjektiv für den Automobilismus in der ersten Hälfte des 20. Jahrhunderts zu finden, denn Moms Thema ist eigentlich ein anderes: Er beschreibt nicht einen, sondern mindestens zwei Automobilismen, die er nicht geographisch, sondern zeitlich unterscheidet.

Das Automobil war in seiner Anfangszeit bis zum Ende des Ersten Weltkriegs - bei Mom der erste Teil des Buches mit dem Titel „Emergence” eine Abenteuermaschine, die Aggressionen und Gewalt ebenso kanalisierte wie provozierte. Die rasenden Fahrten hinaus in eine Umgebung, die man aufgrund der komplizierten Handhabung der Maschine, des Staubs und des Lärms eigentlich kaum wahrnahm, faszinierte vor allem eine auf Gefahr und Distinktion erpichte urbane Elite aus Aristokratie und Bourgeoisie mit einer stark homosozialen, bei Mom bisweilen als machohaft bezeichneten Kultur. In der Zwischenkriegszeit - bei Mom als zweiter Teil mit „Persistence” überschrieben - wandelte sich das Automobil vom „pleasure car” zum „passenger car" (ebd.: 637) Die Mittelklassenfamilie adoptierte das Auto, dessen Gestalt als „affordable family sedan” (ebd.: 638) sich wesentlich von den Abenteuermaschinen der Anfangszeit unterschied. Damit zeigt Mom deutlich auf, was Merriman in seiner kurzen Skizze allzu schnell ineinander schob: Während bei 
Merriman „das Automobil” als quasi ahistorische Größe ein für allemal die ebenfalls eher ahistorisch gedachten menschlichen Wahrnehmungen und Erfahrungen veränderte, unterscheidet Mom sehr genau zwischen unterschiedlichen Nutzergruppen und Nutzungspraktiken des Autos und unterstreicht dessen technische und physische Formbarkeit. Vehement attackiert er zugleich den „toy-to-tool myth”, der den Erfolg des Automobils in seiner Wandlung vom Lustobjekt zum Werkzeug erklären will. Dies sei, so Mom überzeugend, im Wesentlichen eine Marketing-Strategie der Automobilbefürworter gewesen, mit der die Nützlichkeit und die - sich daraus ableitende - gesellschaftliche Notwendigkeit des Autos auch deswegen behauptet wurde, um damit die nach wie vor vorhandenen Lustmomente $\mathrm{zu}$ verschleiern.

Moms Anspruch ist ambitioniert: Er will mit Atlantic Automobilism einen "stepping stone to the field of the so-called New Mobility Studies" schaffen (ebd.: 4). Damit verbindet Mom den Anspruch einer applied history, in dem Sinne, dass er Geschichte nicht nur verstehen, sondern erklären will. Doch gerade weil Mom nicht nur die Frage nach dem „wie”, sondern vor allem nach dem „warum” stellen will, bleibt kritisch zu hinterfragen, ob sein Ansatz einer diachronen Geschichte des Automobils ab 1895 in diesem Zusammenhang sinnvoll ist. Mit diesem langen Zeitraum folgt Mom jenen Studien, die den „holprigen Siegeszug” des Automobils über einen langen Zeitraum zu erklären suchten (Merki 2002). Mom selbst aber macht deutlich, dass der untersuchte Gegenstand, das Automobil, in dieser langen Periode keineswegs immer der gleiche war, sondern dass wir vielmehr sehr genau zwischen unterschiedlichen Formen und Benutzergruppen des Automobils unterscheiden müssen. Denn die Frage nach dem „warum” ist ja selbst keine zeitlose: Sie speist sich aus den Erfahrungen der vom Automobil geprägten Gesellschaften in der Nachkriegszeit und aus den gegenwärtigen Anstrengungen, diesem in vielen Bereichen dominierenden Verkehrsmodus andere Mobilitätsstrategien entgegenzusetzen. Die Tendenz der Historikerinnen und Historiker geht dahin, diese in der Gegenwart dominanten Strukturen mit Hilfe einer langen Geschichte - einer Genealogie heißt es bei Foucault polemisch - zu erklären (Foucault 2009). Foucault wollte hingegen das Augenmerk auf die vergebenen Chancen und abgerissenen Traditionen richten. Ein solcher Blickwinkel ist jedoch eher durch einen synchronen Ansatz zu erreichen.

Es gehört zu den vielen Meriten von Moms Buch, dass er anders als viele seiner Vorgänger nicht nur aufs Automobil blickt, sondern auch auf die vielen anderen Möglichkeiten, mobil zu sein, wie etwa auf das Fahrrad, das Motorrad oder die Eisenbahn. Dennoch steht im Mittelpunkt eben vor allem dieser eine sowohl bei den Nutzergruppen und Nutzungen als auch in seiner technischen und physischen Form durchaus sehr verschieden gestaltete Gegenstand. Moms ausführliche Studie reizt die Grenzen dieser gegenstandsbezogenen, diachronen Betrachtungsweise aufs Äußerste aus und führt gerade dadurch zu 
der Frage, ob nun nicht doch der Zeitpunkt für eine synchrone, nicht auf einen Gegenstand bezogene Mobilitätsgeschichte gekommen ist, eine Geschichte von konkurrierenden Mobilitätsregimes, bei denen es um die Neukonfigurationen von Infrastrukturen, sozialen Ordnungen, Mobilitätskulturen und Mobilitätsmaschinen geht. ${ }^{6}$

\section{Mobilitäten und Materialitäten}

Das stark sozialwissenschaftlich ausgerichtete, aber für historisch orientierte Forscherinnen und Forscher ebenfalls wärmstens zu empfehlende Routledge Handbook of Mobilities entwickelt einen Einstieg in das Feld über unterschiedliche Zugänge. Auch hier widmet sich ein erster Teil den Genealogien, Philosophien und Zugängen, bei denen die unterschiedlichen Mobilitätsansätze in den Disziplinen Geographie, Soziologie, Geschichte und Anthropologie, Migrationsstudien, Tourismusstudien vorgestellt und die Verbindungen mit queer theory und Feminismus erkundet werden. In den folgenden sechs Sektionen präsentieren viele verschiedene Autoren Teilstudien aus den mobility studies, die wiederum in unterschiedliche Zugänge gruppiert sind: Mobilität wird hier anhand von Qualitäten (Sektion 2), Räumen, Systemen und Infrastrukturen (Sektion 3), Materialitäten (Sektion 4), Subjekten (Sektion 5), Ereignissen (Sektion 6) und Methoden (Sektion 7) verhandelt. Namhafte Autoren sind ebenso vertreten wie eher unbekannte, am Anfang der akademischen Karriere stehende Beitragende.

Wie wird nun die diesen Essay leitende Frage nach der Bedeutung der mobility studies für die Verkehrs- und Mobilitätsgeschichte, sowie umgekehrt in diesem Handbuch beantwortet? Colin Divalls Einschätzung in seinem kurzen Überblick zu „Mobilities und Transport History” fällt vorsichtig aus. Divall spart nicht mit Kritik an der bisherigen Verkehrsgeschichte und kritisiert vor allem deren Fixierung auf die unterschiedlichen Verkehrsmodi. Dies führe zu einer hartnäckigen Ignoranz von Ansätzen, die die Rolle der Nutzerinnen und Nutzer in den Mittelpunkt stellen. Zugleich bemängelt er an den mobility studies die fehlende Bereitschaft, sich auf die Objekte und ihre (unterschiedlichen, historischen) Funktionsweisen einzulassen. Bisher sei der Beitrag aus der Verkehrs- und Mobilitätsgeschichte zu den mobility studies eher gering. Allenfalls die Infrastrukturen seien ein willkommener und auf einer langen Forschungstradition basierender Beitrag der Verkehrsgeschichte zu den neueren Ansätzen. Es handele sich um einen Bereich der Verkehrsgeschichte, so Divell, bei dem die politische Bedeutung der materiellen Dimension schon früh evident gewesen sei: Mit Infrastrukturen verbänden sich In- und Exklusionsmechanismen, Räume würden produziert und gegliedert, Machtverhältnisse etabliert (Divall in Adey et al. 2014: 37). 
Interessanterweise enthält auch der eingangs erwähnte Sammelband von Neubert und Schabacher einen einzigen Beitrag, der genau jenen von Divall noch einmal hervorgehobenen, quasi traditionellen Nexus zwischen technischer Infrastruktur und Politikgeschichte herausstellt, nämlich den des Zeithistorikers Dirk van Laak über die Infrastruktur als europäisches Integrationsmedium (van Laak in Neubert, Schabacher 2013: 165-189). Divalls Vorwurf, das Interesse an Objekten und Funktionsweisen sei zu gering, trifft sicherlich nicht auf die Veröffentlichung von Neubert und Schabacher zu. Diese stellen ihrem Sammelband dezidiert einen breiten Medienbegriff voran, mit dem, inspiriert durch Bruno Latours Akteur-Netzwerk-Theorie, der Blick auf Materialitäten und Funktionsweisen gelenkt und technische Funktionsweisen mit politischen und sozialen Dimensionen zusammen gedacht werden sollen.

In ihrem Sammelband vollziehen die Medienwissenschaftler Neubert und Schabacher gemeinsam mit ihren Autorinnen und Autoren genau den material turn, den Colin Divall aus der Perspektive der transport and mobility history einfordert. Als Direktor des Institute of Railway Studies and Transport History in York ist sich Divall auch der ungenutzten Quellen und Methoden dieses material turn bewusst, wenn er auf die große Gruppe von Amateuren im besten Sinne des Wortes verweist, die historische Mobilitätsmaschinen pflegen und über ein weitgehend ungenutztes Wissen über Eigenschaften und Funktionsweisen verfügen (Divall in Adey et al 2014: 41). Eine solchermaßen artefaktbezogene, technikhistorische Forschung mahnte in Deutschland unlängst auch Kurt Möser für die Mobilitätsgeschichte an (Möser 2014). Wie das Dilemma zu lösen ist, gegenwärtige Nutzungserfahrungen in angemessene Relation zu den historischen Nutzungserfahrungen und -kontexten zu stellen, vermag Möser indes nicht zu sagen. Trotz des wiederkehrenden Rufs nach mehr objektbezogener Forschung bedauert Divall in seinem Beitrag zugleich, dass die Verkehrs- und Mobilitätsgeschichte zu stark auf individuelle Mobilitäten fokussiere und der Blick auf Systeme und auf Waren- und Gütertransporte weitgehend fehle (Divall in Adey et al. 2014: 40).

\section{Anstelle eines Fazits: Mobilitätsgeschichte „on the move”}

Die Rhetorik des „[being] on the move” ist in den mobility studies allgegenwärtig und dominiert auch viele der hier diskutierten Studien von Vertreterinnen und Vertretern der verschiedensten wissenschaftlichen Disziplinen. Es sollen (disziplinäre) Grenzen eingerissen und neue Sprungbretter entwickelt werden. Deshalb sei es der Rezensentin verziehen, dass sie 
angesichts der hehren Ziele doch zu einem eher zurückhaltenden Ergebnis kommt: Was bedeuten sie nun, die new mobility studies?

Die Antwort ist - fast unweigerlich -, dass sie viel Unterschiedliches für unterschiedliche Disziplinen bedeuten. Mobilität beinhaltet eine soziale ebenso wie eine technisch-materielle, eine räumliche genauso wie eine zeitliche Dimension. In den wenigsten Fällen lassen sich diese unterschiedlichen Aspekte fein säuberlich unterscheiden. In diesem Essay wurden vor allem Studien aus Disziplinen berücksichtigt, die den Schwerpunkt ihrer traditionellen Ausrichtung auf einen dieser Aspekte legen. Dabei ist der Grad der wissenschaftlichen Mobilisierung durch den Begriff Mobilität zutiefst unterschiedlich. In der Soziologie hatten Urry und Sheller den "mobility turn” analog zu den verschiedenen „turns” in den Kulturwissenschaften ausgerufen, um das analytische Korsett „Gesellschaft” zu sprengen. In der Humangeographie sollen mit mobility die Kategorien Zeit und Raum ausgehebelt werden. Mimi Sheller konstatiert in ihrem Beitrag zum Handbook of Mobilities selbst ein wenig ernüchtert, „mobility” sei als Konzept vor allem bei den (HumanGeographen auf fruchtbaren Boden gestoßen, in der Soziologie sei der Begriff hingegen weiterhin stark sozial und eben nicht räumlich und technisch belegt (Sheller in Adey et al. 2014: 45).

Als Schlagwort wendet sich Mobilität auch gegen bestimmte Forschungstraditionen und -richtungen innerhalb der jeweiligen Fachdisziplinen. Wie anfänglich anhand von Neubert und Schabachers kurzer Begriffsgeschichte erläutert, hat der Begriff Verkehr ein weites, durchaus auch schillerndes und vielfältig auslegbares Bedeutungsfeld. Zugleich hat Anette Schlimms Studie jedoch deutlich gemacht, dass sich in der Mitte des 20. Jahrhunderts mit dem Begriff Verkehr bestimmte Vorstellungen von „social engineering und sozialen Ordnungen" (Schlimm 2011: 301-310) verbanden, die ihn für die heutige Zeit wenig attraktiv machen. Fast scheint es so, als sei ein Großteil der jetzigen Mobilitätsgeschichte noch immer damit beschäftigt, sich von den gestrengen Vorvätern der Verkehrsgeschichte zu emanzipieren, die den Verkehr in „nützlich” und „unnütz” unterteilten und eine tiefe Skepsis gegenüber der Relevanz individueller Mobilitätserfahrungen hegten. Die Mobilitätsgeschichte will die Nutzerinnen und Nutzer nicht weiter entmündigen, sondern sieht sie vielmehr als zentralen Faktor innerhalb der verschiedenen Mobilitätsregimes, sie betrachtet die in der Interaktion zwischen Mensch und Maschine entstehenden Erlebnisse und Wahrnehmungen ebenso wie die sozialen und kulturellen Kontexte, innerhalb derer diese Erfahrungen gleichermaßen konstruiert werden.

Gegenüber den anderen, vielfach sozialwissenschaftlich geprägten Ansätzen in den mobility studies hat die aus der Verkehrsgeschichte hervorgehende historische Forschung einerseits eine lange Tradition in der genauen Betrachtung materieller Kultur und technischer Funktionsweisen, die in den 
neueren Ansätzen aus der Medientheorie oder der Akteur-Netzwerk-Theorie ebenfalls relevant sind. Mit ihrer starken Tradition in diachronen Ansätzen droht sich die Mobilitätsgeschichte vielleicht aber auch ein Bein zu stellen: Der Blick auf einen Gegenstand verleitet zu einem langen Untersuchungszeitraum, umgekehrt lässt sich die lange Zeitspanne leichter über ein Objekt in den Blick bringen. Hier könnte ein wichtiger Impuls aus den mobility studies sein, den Blick stärker auf synchrone Vorgänge zu konzentrieren und damit die Fixierung auf das Automobil oder das Fahrrad zugunsten eines Blickes auf unterschiedliche Mobilitätskulturen und -regimes $\mathrm{zu}$ verschieben. Bislang fehlt anscheinend vielfach immer noch der Mut, von den verschiedenen Benutzergruppen, Nutzungsweisen und Mobilitätsmaschinen ausgehend allgemeinere Aussagen über Mobilitätsregimes zu tätigen.

Zugleich verdeutlichen Studien wie jene von Mimi Sheller, wie schwierig es ist, Mobilität in ihren komplexen globalen Zusammenhängen und auf den unterschiedlichen Ebenen von Produktion und Konsumtion, realer und symbolischer Mobilität, in den analytischen Griff zu bekommen. Unabdingbar ist der Blick auf Mobilität und Immobilität gleichermaßen, um nicht in den Allgemeinplatz ubiquitärer Mobilität oder gar in eine einseitige Fortschrittsgeschichte ständig zunehmender Mobilität zu verfallen.

Schließlich aber gibt es noch den berühmten „elephant in the room” in Hinblick auf den von Gijs Mom und Colin Divall gleichermaßen eingebrachten Ansatz einer usable past, einer für die heutige Politik nutzbar gemachten historischen Aufarbeitung. Mit dem Schlagwort mobility studies verbindet sich nicht zuletzt ein Programm zur Allokation von Forschungsmitteln und zur akademischen Institutionalisierung. Der Anspruch dieser historisch orientierten Vertreter der new mobility studies, auch als Verkehrsund Politikberatung agieren zu können, steht jedoch bislang weitestgehend uneingelöst im Raum. Es erscheint höchst zweifelhaft, ob es überhaupt möglich ist, eine die Bedürfnisse der Politikberatung unmittelbar erfüllende Mobilitätsgeschichte zu schreiben, die gleichzeitig multiperspektivisch auf den verschiedenen Ebenen von Produktion und Konsumtion operiert und technisch-physische ebenso wie symbolische und soziokulturelle Aspekte berücksichtigt.

Die Überwindung der nationalen Betrachtungsgrenzen, die Neu-Orientierung weg vom einseitigem Blick auf die Produktion hin zur Konsumtion, die Ergänzung der großen diachronen Analysen eines Verkehrsträgers, mit dem bisher die Vielfältigkeit und Formbarkeit der Mobilitätskulturen und -maschinen verdeutlicht wurden, durch einen noch stärkeren Blick auf synchrone Entwicklungen über die unterschiedlichsten Verkehrsträger und Mobilitätskulturen hinweg: All dies sind Herausforderungen, vor denen die Mobilitätsgeschichte aufgrund der interdisziplinären Diskussionszusammenhänge der mobility studies steht. Dieser mittlerweile fest etablierte, 
interdisziplinäre Kontext wird dafür sorgen, dass die Verkehrs- und Mobilitätsgeschichte auch in Zukunft „on the move” bleibt.

\section{Anmerkungen}

1 Ein regelmäßiger Kommentator der Forschungsdiskussionen im Bereich der mobility history ist Gijs Mom, auf dessen Artikel zur Situation beim Journal of Transport History (Mom 2003) hier stellvertretend verwiesen sei.

$2 \mathrm{Zu} \mathrm{t2m} \mathrm{siehe} \mathrm{deren} \mathrm{Homepage:} \mathrm{http://t2m.org.} \mathrm{Abruf} \mathrm{vom} \mathrm{7.} \mathrm{April} 2015$.

3 Ein Überblick über die internationalen Organisationen zur Verkehrsgeschichte und Mobilität findet sich auch bei Dienel and Divall (2009).

4 Gänzlich entgangen ist ihm etwa das Baseler Forschungsprojekt zu material flows, siehe http://material-flow.ch/de/.

5 Hervorzuheben wären hier neben den bereits oben genannten Cresswell (2006), Massey (2005) und Thrift (2006).

6 Kurt Mösers Habilitationsschrift über Kulturen individueller Mobilitätsmaschinen 1880-1930 hatte einen solchen Ansatz angedacht, allerdings ebenfalls viel zu einseitig in Richtung eines diachronen Siegeszug von den Anfängen in die Gegenwart konstruiert. Vgl. Möser 2009.

\section{Literatur}

Cresswell, Tim 2006. On the Move. Mobility in the Modern Western World. London und New York: Routledge.

Dienel, Hans-Liudger und Colin Divall 2009. Changing Histories of Transport and Mobility in Europe. In: Roth, Ralf und Karl Schlögel (Hg.), Neue Wege in ein neues Europa. Geschichte und Verkehr im 20. Jahrhundert. Frankfurt am Main: Campus, 65-84.

Dommann, Monika 2012. Rezension zu Richard Vahrenkamp: Die logistische Revolution. Der Aufstieg der Logistik in der Massenkonsumgesellschaft. Frankfurt am Main 2011, H-Soz-uKult 24.10.2012.

Foucault, Michel 2009. Nietzsche, die Genealogie, die Historie. In: Ders.: Geometrie des Verfahrens. Schriften zur Methode. Herausgegeben von Daniel Defert und Francois Ewald unter Mitarbeit von Jacques Lagrange. Berlin: Suhrkamp, 181-205.

Hannam, Kevin, Mimi Sheller und John Urry 2006. Mobilities, Immobilities and Moorings. Mobilities 1,1, 1-22.

Massey, Doreen 2005. For Space. London: Sage.

Merki, Christoph Maria 2002. Der holprige Siegeszug des Automobils 1895-1930. Zur Motorisierung des Straßenverkehrs in Frankreich, Deutschland und der Schweiz. Wien: Böhlau.

Merki, Christoph Maria 2008. Verkehrsgeschichte und Mobilität. [UTB Profile] Stuttgart: Eugen Ulmer Verlag.

Mintz, Sydney W. 1985. Sweetness and Power: The Place of Sugar in Modern History. New York: Penguin Viking Books.

Mom, Gijs 2013/2014. Transmodal, transnational, transdisziplinär: Die Krise der Geschichte des Transportwesens und Wege zu deren Behebung. Blätter für Technikgeschichte 75/76, 27-46.

Mom, Gijs 2003. What Kind of Transport History Did We Get? Half a Century of JTG and the Future of the Field. Journal of Transport History 24, 121-138.

Möser, Kurt 2009. Fahren und Fliegen in Frieden und Krieg: Kulturen individueller Mobilitätsmaschinen 1880-1930. [= Technik + Arbeit: Schriften des Landesmuseums für Technik und Arbeit in Mannheim 13] Heidelberg: Verlag Regionalkultur. 
Möser, Kurt 2014. Transport-, Verkehrs- oder Mobilitätsgeschichte? Neue Paradigmen der Technik- und Industriekultur. In: Henry Keazor, Dominik Schmitt, Nils Daniel Peiler (Hg.), Genialer Schrott. Interdisziplinäre Studien zur Industriekultur. Saarbrücken: universaar, 61-82.

Sheller, Mimi und John Urry 2006. The New Mobilities Paradigm. Environment and Planning A 38, 207-226.

Thrift, Nigel 2006. Space. Theory, Culture and Society 23, 139-155.

Urry, John 2000. Sociology Beyond Societies. Mobilities for the Twenty-first Century. [International Library of Sociology] London und New York: Routledge.

Urry, John 2007. Mobilities. Cambridge und Malden, MA: Polity Press.

Urry, John 2010. Mobile Sociology. The British Journal of Sociology 61, 347-366.

\section{Anne-Katrin Ebert}

Technisches Museum Wien

Mariahilfer Str. 212

1140 Vienna

Austria

E-Mail: anne.ebert@tmw.at 